\title{
L'ECOLOGIA DEL PICCHIO NERO, DRYOCOPUS MARTIUS, NELLE FORESTE CASENTINESI COME CHIAVE PER LA POSSIBILE ESPANSIONE DELLA SPECIE NELL'APPENNINO
}

\begin{abstract}
Riassunto - I modelli matematici rappresentano utili strumenti per la definizione della distribuzione e delle preferenze ecologiche delle specie animali, assumendo un ruolo di primo piano nella definizione delle politiche di conservazione. Tra i numerosi metodi a disposizione, recentemente hanno avuto particolare sviluppo quelli che utilizzano dati di sola presenza; tra questi particolarmente efficace risulta MaxEnt. La recente colonizzazione da parte del Picchio nero del Parco Nazionale delle Foreste Casentinesi offre un caso di studio particolarmente interessante per presentare questo metodo di analisi, indagando le possibili evoluzioni di un processo di espansione tuttora in corso.
\end{abstract}

Parole chiave - Dryocopus martius, MaxEnt, Parco Nazionale Foreste Casentinesi, Appennino settentrionale.

Abstract - Ecological mathematical models are useful tools to define geographic distribution and ecological features of species, playing a fundamental role in defining conservation policies. Through the various methods we can use, recently, those which work with only-presence-data are growing in popularity and particularly MaxEnt. The recent colonization of Foreste Casentinesi National Park by Black Woodpecker represent a very interesting study-case to show how this method work, analysing possible evolution of an expansion process that is still evolving.

Key words - Dryocopus martius, MaxEnt, Foreste Casentinesi National Park, Northern Apennines.

\section{Introduzione}

La conoscenza della distribuzione e delle esigenze ecologiche delle specie costituisce un prerequisito fondamentale per qualsiasi azione di conservazione o gestione (RUSHTon et alii, 2004) e in questo ambito i modelli

* DREAM Italia - Via Garibaldi, 3 - 52015 Pratovecchio (AR). - E-mail: emberiza1978@gmail.com

** STERNA - Via Giuseppe Pedriali, 12 - 47121 Forlì.

**** Parco Nazionale Foreste Casentinesi, Monte Falterona e Campigna - Via Nefetti, 3 - 47018 Santa Sofia (FC). 
matematici rappresentano strumenti molto utili per ottenere questo tipo di informazioni (BRotons et alii, 2004). Sebbene siano numerosi i metodi a disposizione (Guisan \& Zimmerman, 2000; Burgman et alii, 2005), negli ultimi anni, hanno acquisito sempre maggiore importanza quelli che impiegano dati di sola presenza (Elith et alii, 2006). L'impiego di queste metodologie ha un indubbio vantaggio: molti autori hanno evidenziato come analisi condotte su dati di presenza-assenza possano portare ad una erronea valutazione dei parametri ambientali che influiscono sulla distribuzione di una specie (Mollanen, 2002; Gu \& Swihart, 2004). Ł̀ infatti ben noto come l'assenza sia, ad eccezione di alcuni casi, difficile da accertare, soprattutto quando si ha a che fare con specie rare o dal comportamento criptico che, soprattutto nel primo caso, rappresentano spesso quelle di maggiore interesse nel campo della biologia della conservazione (KERR et alii, 2000).

Tra i metodi che impiegano dati di sola presenza ha trovato recente impiego il programma MaxEnt (Phillips et alii, 2006; Phillips \& Dudík, 2008) che, utilizzando le informazioni ambientali disponibili per i punti di presenza della specie confrontate con quelle dell'intero territorio (punti "background"), restituisce una distribuzione spaziale dell'idoneità del territorio per la specie stessa (RAes \& TER STEege, 2007). Il metodo, basato sul principio di massima entropia, individua appunto la distribuzione a entropia massima, ovvero quella che, tenendo conto della natura dei fattori ambientali che caratterizzano sia i punti di presenza che l'intera area di studio, utilizzati come "costrittori", si avvicina maggiormente ad una distribuzione uniforme (PHILlips et alii, 2006).

Confrontato con altri metodi disponibili, MaxEnt sembra particolarmente efficiente ed affidabile (Elith et alii, 2006; Peterson et alii, 2007; GuIsan et alii, 2007), riuscendo a dare risultati utili anche con campioni di numerosità limitata (PAPE \& GaUbert, 2007; PEARson et alii, 2007) e permettendo di considerare relazioni di differente tipo tra presenza della specie e variabili ambientali (lineari, curvilinee o complesse; ELith et alii, 2006). I risultati ottenuti con questo metodo sono inoltre meno influenzati da livelli ineguali di campionamento, caratteristica che, in considerazione della poca omogeneità dei dati in genere disponibili a scala vasta, spesso concentrati in aree particolari come tipicamente le aree protette, può essere molto utile per scopi applicativi come l'identificazione delle aree di maggior interesse per la conservazione (TsOAR et alii, 2007).

Un altro indubbio vantaggio è la possibilità di utilizzare dati raccolti in maniera non standardizzata, tra cui, solo per fare alcuni esempi, reperti e segnalazioni contenute in musei o collezioni, o database di progetti a vasta scala che non prevedono metodologie di censimento definite, di cui oggi c'è una crescente disponibilità (cfr. Ornitho; LARDELLI, 2009). Queste banche dati risultano spesso particolarmente interessanti, soprattutto quando si ha a che fare con specie rare, e possono contribuire non poco a 
incrementare il numero di dati disponibili.

L'utilizzo di MaxEnt pone naturalmente anche alcuni problemi, che comunque, se debitamente considerati, non sembrano inficiare i risultati finali delle analisi. In particolare, MaxEnt utilizza un modello esponenziale per il calcolo delle probabilità e quindi non ha un limite superiore per questi valori; questo può determinare, nel caso si vadano ad "esportare" i risultati in altre aree, caratterizzate da condizioni ambientali che non ricadono nel range dell'area di studio, valori di probabilità molto alti, non riconducibili ad un corrispondente livello di idoneità ambientale ("overfitting"; Phillips et alii, 2006). Per questo motivo, qualora ci si trovi in queste condizioni, è importante limitare l'esportazione dei modelli a aree con caratteristiche ambientali paragonabili, individuando "un'area di calibrazione" (FicETola et alii, 2007).

Recentemente il metodo è stato impiegato in Italia in alcuni studi ecologici riguardanti sia uccelli (Tellini Florenzano et alii, 2008; CAMPEDELLi et alii, 2009; Cutini et alii, 2009) che altri taxa (rettili, FicETOLA et alii, 2008; Bомві et alii, 2009; farfalle, FAviLLI et alii, 2008; pipistrelli, AGNELLI et alii, 2009), mostrando di poter offrire un'utile modalità di analisi di dati raccolti con differenti metodologie. L'obiettivo principale di questo lavoro è quello di presentare, attraverso un esempio pratico, un metodo di analisi ecologica che, nonostante presenti indubbi vantaggi ed un campo di applicazione assai vasto, risulta ancora poco diffuso in Italia.

La popolazione di Picchio nero, Dryocopus martius, nel Parco Nazionale delle Foreste Casentinesi, nell'Appennino settentrionale si presenta, a questo proposito, come un caso di studio molto interessante (CECCARELLI et alii, 2008), sia da un punto di vista gestionale, sia da un punto di vista squisitamente conoscitivo, fornendo spunti interessanti per cercare di capire la possibile evoluzione di un fenomeno dinamico tuttora in corso.

Il Picchio nero in Italia è distribuito prevalentemente nelle regioni settentrionali, dove occupa, praticamente senza soluzione di continuità, tutto l'arco alpino, con presenza in alcuni settori dell'Appennino meridionale, dove però le segnalazioni, soprattutto recenti, risultano lacunose e non permettono di definire in maniera adeguata l'areale della specie (Brichetti \& Fracasso, 2007; Pedrini, 2010). Come conseguenza di un significativo incremento delle popolazioni alpine e prealpine (BRICHETTI $\&$ FrACASSO, 2007), processo che si inserisce in un più vasto fenomeno registrato oramai da diversi anni a livello europeo (MIKUSINSKI, 1995; BURFIELD \& VAN BOMMEL, 2004), il Picchio nero mostra una evidente tendenza ad espandere il proprio areale verso quote più basse, collinari e addirittura di pianura, dapprima limitata al periodo invernale (Mezzavilla \& Stival, 1996; Semenzato \& Amato, 1998; Parodi, 1999; Utmar, 2003) e successivamente registrata anche in quello riproduttivo. Già a partire dai primi anni del nuovo millennio si è assistito ad un incremento costante delle segnalazioni in pianura, anche di nidificazione accertata, in tutte 
le regioni settentrionali; dettagli a livello di singole regioni sono riportati per il Piemonte (Bordignon, 2004; Aimassi \& Reteuna, 2007), Lombardia (Gagliardi et alii, 2007; Pirovano, 2009), Veneto (Mezzavilla, 1989; Martignago et alii, 1992 e 2001; Mezzavilla et alii, 1999; Mezzavilla \& Bettiol, 2007) e Friuli Venezia Giulia (Utmar \& Padovan, 2005; Tofful \& SPONZA, 2007 e 2010).

Nel quadro di questo fenomeno espansivo, in cui potrebbe inserirsi anche il recente rinvenimento, in periodo riproduttivo, nell'Appennino ligure ad est di Genova (BAGHINo, 2009), si colloca la scoperta della specie nel Parco delle Foreste Casentinesi (CECCARELli et alii, 2003), dove la locale popolazione, con ogni probabilità insediatasi non prima del 2000 e stimata alla fine del 2008, ultimo anno in cui sono stati raccolti i dati utilizzati in questo studio, in 4-5 coppie (CECCARELLI et alii, 2008; CECCARELLI, 2011), conta attualmente non meno di 6-7 coppie (P.P. Ceccarelli in verbis). A partire dai dati di presenza di questa popolazione, abbiamo indagato le caratteristiche ecologiche della specie come strumento per comprendere anche le possibili dinamiche di origine e di espansione della popolazione sia a scala locale (cioè nel territorio del Parco) sia di intera penisola.

\section{Materiali e metodi}

I dati di presenza del Picchio nero utilizzati per la costruzione dei modelli sono stati raccolti durante gli anni 2000-2008, all'interno del Parco Nazionale delle Foreste Casentinesi (da ora indicato come "Parco"), e riguardano sia osservazioni dirette $(n=74)$ che localizzazioni di fori di alimentazione e nidi (in seguito definiti "segni"; $n=168$ ). I modelli sono stati costruiti testando l'effetto di alcune variabili ambientali sulla probabilità di presenza della specie, e precisamente: presenza e estensione di alcune tipologie forestali particolarmente idonee alla specie, ovvero l'abetina (Abt2000) e la faggeta (Fgt2000), e due fattori di tipo climatico, il termotipo (Ter) e l'ombrotipo (Omb). Per quanto riguarda le tipologie forestali abbiamo selezionato, rispettivamente per l'abetina e la faggeta, i codici 3.1.2.3 3.1.3.2.3 e 3.1.1.5, 3.1.3.1.5 dalla carta Corine Land Cover (BosSARD et alii, 2000), calcolando la copertura, espressa in percentuale, in un intorno circolare di 2000 metri di raggio da ciascun punto. Questa scala è stata scelta in base alle informazioni disponibili in letteratura sull'ecologia della specie e sull'estensione dei suoi territori (GoRMAN, 2004).

Per quanto riguarda i fattori climatici, abbiamo utilizzato la suddivisione dell'Italia proposta da BLASI et alii (2004) in base alle temperature (il termotipo che è definito da un indice dato dalla somma di temperatura media annua, media delle temperature massime del mese più freddo $\mathrm{e}$ media delle temperature minime del mese più freddo) e in base alle precipitazioni (l'ombrotipo che è definito dal rapporto tra la somma delle pre- 
cipitazioni dei mesi con temperatura media superiore a $0^{\circ} \mathrm{C}$ e la somma delle temperature degli stessi mesi).

Disponendo, per il solo territorio del Parco, di una carta dettagliata sulla vegetazione (VICIANI \& AGOSTINI, 2008) abbiamo voluto verificare se l'utilizzo di informazioni a scala locale influenzasse il potere predittivo del modello. Abbiamo quindi definito una variabile di tipo categoriale utlizzando la tipologia forestale all'interno del quale ricadevano i punti (TypeF). Questa variabile è stata costruita associando dei codici numerici, scelti casualmente, alle diverse tipologie vegetazionali presenti: 0 non bosco; 1 faggeta alta (di crinale); 2 faggeta bassa; 3 abetina; 4 altre conifere e 9 altre latifoglie. Tutte le informazioni sono state associate ai punti di presenza e di background (10201 punti) in ambiente GIS.

Utilizzando MaxEnt (versione 3.1.0.) abbiamo costruito quattro modelli: due per le osservazioni, utilizzando in un caso le quattro variabili generali, estensione di abetina e faggeta, termotipo e ombrotipo (modello "osservazioni") e in un altro caso utilizzando anche la variabile locale, TypeF (modello "osservazioni Parco") e, allo stesso modo, due per i segni (modello "segni" e modello "segni Parco"). I due modelli costruiti senza la variabile TypeF, che è definita solo all'interno dei confini del Parco, sono stati esplicitati anche a livello di Italia peninsulare, utilizzando un layout di esplicitazione composto da 52347 punti, distanziati tra loro circa $2 \mathrm{~km}$, a cui erano stati precedentemente associati gli stessi parametri utilizzati nella costruzione dei modelli. L'esplicitazione dei modelli è stata effettuata solo all'interno di quella che viene definita area di calibrazione (FICETOLA et alii, 2007), ovvero solo all'interno di quelle zone in cui le variabili ambientali utilizzate nel modello assumono valori compresi nel range che caratterizza l'area in cui il modello è stato costruito, cioè il Parco. Per definire l'area di calibrazione si sceglie tra le variabili uno o più fattori di calibrazione; in questo caso è stato utilizzato l'ombrotipo. L'utilizzo di un'area di calibrazione si rende necessario per i problemi di overfitting già discussi in sede di introduzione.

Una volta esplicitati i modelli occorre selezionare dei valori soglia che permettano di individuare quelli che sono effettivamente i territori più idonei alla specie. Il programma fornisce diverse soglie e la loro scelta può apparire non sempre immediata (cfr. LiU et alii, 2005); noi abbiamo selezionato due opzioni, la prima (Minimum Training Presence), che si può definire la più conservativa (PEARson et alii, 2007), prevede di identificare le aree idonee in base al valore minimo di idoneità, tra quelli corrispondenti ai dati effettivi; la seconda (Maximum Training Sensitivity plus Specificity), analiticamente determinata (HERNANDEZ et alii, 2006), corrisponde al valore di idoneità per il quale si minimizzano sia gli errori di omissione ("false presenze"), sia quelli di esclusione ("false assenze"). Le aree individuate in base a questi valori sono state cartografate.

Abbiamo inoltre approfondito, allo scopo di fornire un ulteriore stru- 
mento di utilizzo del programma, un aspetto che riguarda i differenti tipi di relazioni con le variabili, lineari, quadratiche e complesse, tra cui le relazioni di interazione (product), soglia (threshold) e lineare complessa (hinge) che MaxEnt permette di considerare. Le relazioni di tipo hinge sono relazioni di tipo lineare, con la particolarità che assumono un valore costante al di sotto di una certa soglia. L'utilizzo di queste ultime richiede particolare attenzione poiché i risultati possono portare a errate valutazioni sull'importanza di alcune variabili e soprattutto sull'esistenza di valori soglia ecologicamente poco coerenti e difficilmente spiegabili. Il programma permette di modulare l'effetto di queste relazioni utilizzando il coefficiente di regolazione (PHILLIS \& Dudík, 2008), un numero compreso tra 1 e 3 , regolabile manualmente direttamente dalla finestra di dialogo del programma; sostanzialmente, valori più alti corrispondono relazioni più smussate e sovente più facilmente interpretabili da un punto di vista ecologico. Vista l'importanza centrale di questo argomento, abbiamo ritenuto opportuno, a titolo di esempio e per il solo modello dei segni, illustrare e discutere i differenti risultati ottenuti utilizzando varie combinazioni di tipologie di relazioni (features) e di valori del coefficiente di regolazione.

Per la valutazione dell'efficienza dei modelli ottenuti, abbiamo utilizzato il valore dell'area sotto la curva ROC (AUC; FIELDING \& BELL, 1997). L'AUC può assumere valori che vanno da 0,5 (modello senza nessuna capacità predittiva) a 1 (modello che prevede esattamente la presenza della specie; Hosmer \& Lemenshow, 2000) e può essere utilizzata per confrontare l'efficienza di più modelli, indipendentemente dalle dimensioni del campione. Per valutare se l'utilizzo di variabili ambientali calcolate a scala locale aumenta l'efficacia del modello sono stati utilizzati tre parametri: la AUC e le due stesse soglie utilizzate per la definizione degli areali, considerando come migliore il modello maggiormente conservativo, ovvero capace di individuare in maniera più ristretta l'area a maggiore idoneità ambientale (Pearson et alii, 2007).

Nel corso delle analisi non abbiamo tenuto conto dell'effetto dell'autocorrelazione spaziale poiché, a differenza della distribuzione reale, quella potenziale, ovvero individuata mediante l'elaborazione di modelli ecologici, non è influenzata da correlazioni di tipo spaziale e non è quindi necessario tenerne di conto durante le analisi (PHiLLIPs et alii, 2006).

\section{Risultati}

I quattro modelli elaborati hanno tutti valori di AUC elevati, ben superiori alla soglia di 0,8 (Tabella I). Per quanto riguarda l'effetto relativo delle singole variabili si evidenzia una certa differenza tra i risultati ottenuti con le osservazioni rispetto a quelli ottenuti con i segni. 
Tab. I - Elenco delle variabili risultate nei quattro modelli; per ciascuna è indicato il contributo percentuale all'interno di ciascun modello. I modelli che compaiono con la dizione "Parco" sono stati costruiti utilizzando anche la variabile TypeF, ovvero le tipologie forestali desunte dalla carta della vegetazione del Parco. Viene inoltre indicato il valore AUC.

Tab. I - List of the variables entered in the the four models: two of them build using direct observation data (osservazioni) and the other two indirect presence signs only (segni). For each variable the percentage contribution is reported. Models signed with the code "Parco" are those built using the Type F variable, that is the various forest typologies deduced by the Foreste Casentinesi National Park vegetation map. For each model AUC is also reported.

\begin{tabular}{lcccccc}
\hline modello & Abt 2000 & Fgt 2000 & Omb & Ter & TypeF & AUC \\
\hline Osservazioni Parco & 16,6 & 5,3 & 39,4 & 13,9 & 24,8 & 0,926 \\
Osservazioni & 20,2 & 16,3 & 45,6 & 17,9 & - & 0,909 \\
Segni Parco & 15,4 & 13,9 & 11,6 & 20,1 & 38,9 & 0,917 \\
Segni & 29,3 & 27,6 & 14,9 & 28,2 & - & 0,904 \\
\hline
\end{tabular}

Nel primo caso è la variabile ombrotipo a fornire il contributo maggiore, mentre le altre tre variabili contribuiscono con valori simili comparabili tranne la faggeta che, nel modello costruito utilizzando anche le tipologie forestali del Parco, assume un valore molto basso, probabilmente proprio a causa dell'interazione con la variabile TypeF, il cui contributo è consistente. Le relazioni con le due variabili climatiche hanno sempre un andamento lineare, con valori crescenti verso condizioni climatiche più fresche e umide e caratterizzate da valori di temperature più bassi. Anche la relazione con l'abetina risulta lineare con valori crescenti al crescere della percentuale di copertura; diversa invece la relazione con la faggeta, in questo caso infatti, il modello sembra ben individuare un punto di massimo, corrispondente all'incirca ad una copertura del 50-60\%, oltre il quale l'effetto della faggeta diventa negativo (Figura 1).

a) $\mathrm{Abt} 2000$

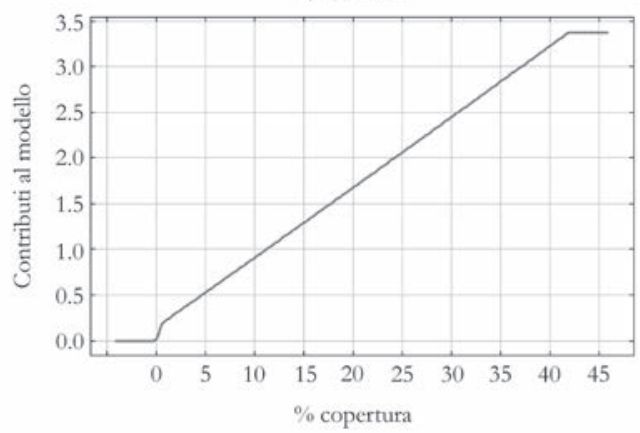

b) Fgt2000

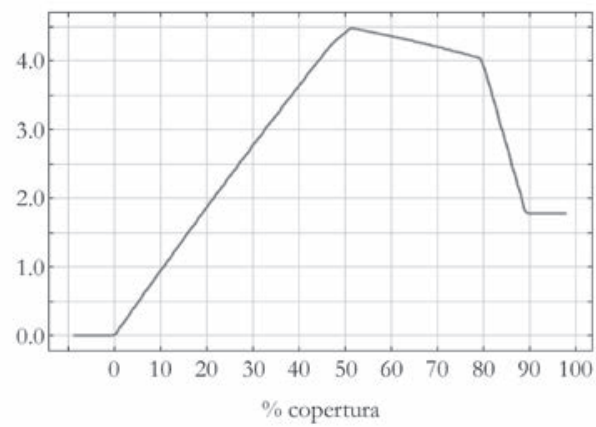

Fig. 1 - Andamento della relazione tra probabilità di presenza del Picchio nero, calcolata utilizzando le sole osservazioni, e copertura percentuale di abetina (a) e faggeta (b).

Fig. 1 - Relationship between the probability of Black Woodpecker presence, estimated using observation data, and the coverage, expressed as percentage, of silver fir (a) and beech (b) woods. 
Per quanto riguarda invece i modelli elaborati a partire dai segni di presenza, il termotipo contribuisce in maniera decisamente superiore rispetto al modello precedente, assumendo un'importanza maggiore anche rispetto all'ombrotipo; anche in questo caso le variabili faggeta e abetina assumono valori simili e comunque decisamente più bassi quando nel modello si inserisce anche la variabile TypeF. Le tipologie delle relazioni esistenti tra segni di presenza e variabili ambientali sono del tutto analogghe a quella descritta per i due modelli precedenti; è interessante notare come, anche in questo caso, sebbene per valori di copertura leggermente maggiori, il modello individua un massimo oltre il quale l'estensione della faggeta assume un effetto negativo (Figura 2).

a) $\mathrm{Abt} 2000$

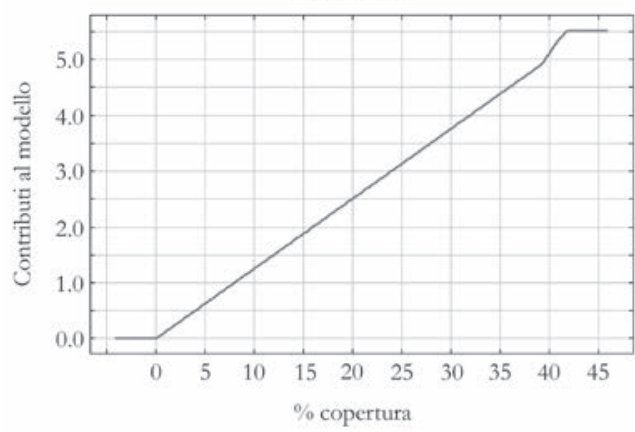

b) Fgt2000

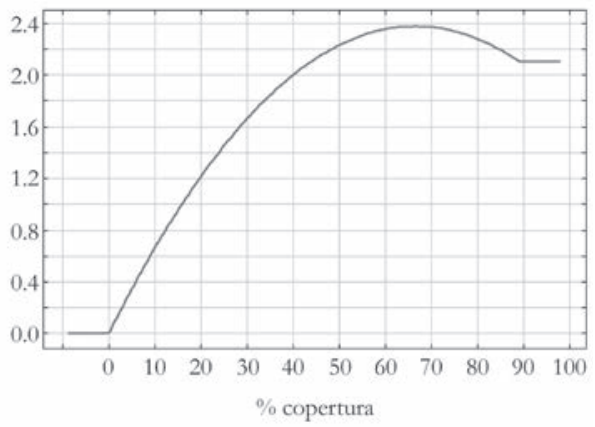

Fig. 2 - Andamento della relazione tra probabilità di presenza del Picchio nero, calcolata utilizzando solo i segni di presenza, e la copertura percentuale di abetina (a) e faggeta (b).

Fig. 2 - Relationship between the probability of Black Woodpecker presence, estimated using indirect presence data (e.g. nesting hole), and the coverage, expressed as percentage, of silver fir (a) and beech (b) woods.

Nelle Figure 3 e 4 vengono esplicitati i valori di idoneità ambientale a livello di Parco ottenuti dai due modelli senza la variabile TypeF; sono riportate anche le localizzazioni dei dati reali.

L'utilizzo di parametri ambientali locali, quindi con una scala di dettaglio maggiore, e migliore, permette effettivamente di incrementare l'efficacia del modello; in entrambi i casi infatti, ai modelli in cui è stata inclusa anche la variabile TypeF sono associati valori di AUC superiori ed entrambe le soglie individuano percentuali di territorio idoneo inferiori (Tabella II). 
Tab. II - Parametri utilizzati per confrontare l'efficacia dei modelli costruiti utilizzando anche la variabile TypeF, quelli che compaiono con la dizione Parco, con quelli in cui questa variabile non compare. Per ciascun parametro è riportato

il coefficiente fornito dal modello; per MTP (Minimum Training Presence)

e MTSS (Maximum Training Sensitivity plus Specificity) è riportata la percentuale di territorio idoneo individuato dalle soglie.

Tab. II - Parameters used to compare models build with local environmental variables (signed with the code "Parco") with those without.

For each variables is indicated the coefficient resulted from the models; for MTP (Minimum Training Presence) e MTSS (Maximum Training Sensitivity plus Specificity) the percentage of suita.

\begin{tabular}{lccc}
\hline modello & AUC & MTP & MTSS \\
\hline Osserv. Parco & 0,926 & 0,326 & 0,210 \\
Osserv. & 0,909 & 0,350 & 0,274 \\
Segni Parco & 0,917 & 0,335 & 0,172 \\
Segni & 0,904 & 0,413 & 0,187 \\
\hline
\end{tabular}
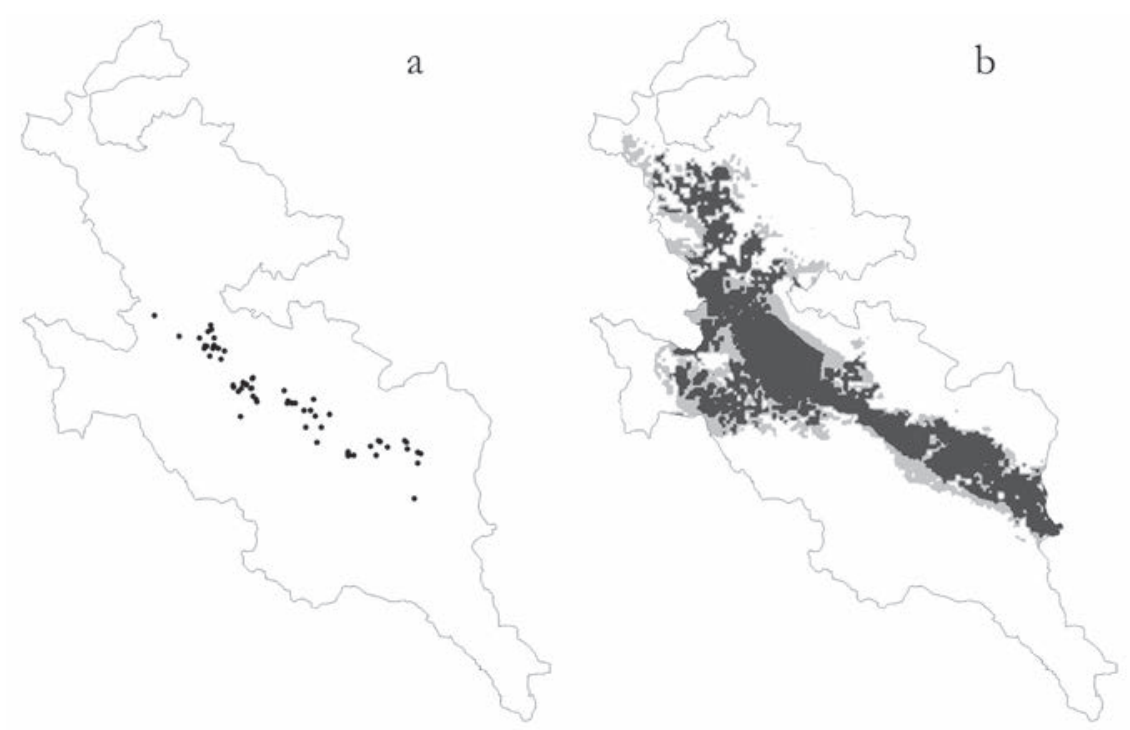

Fig. 3 - Carte delle osservazioni dirette (a) e dell'idoneità ambientale (b) per il Picchio nero nel Parco Nazionale delle Foreste Casentinesi, i cui valori si riferiscono al solo modello costruito con le osservazioni dirette; è riportato il confine dell'area protetta.

Fig. 3 - Maps of the observations (a) and of habitat suitability (b) for the Black Woodpecker in the Foreste Casentinesi National Park; values are relative only to the model built with observations. The National Park border is also reported. 

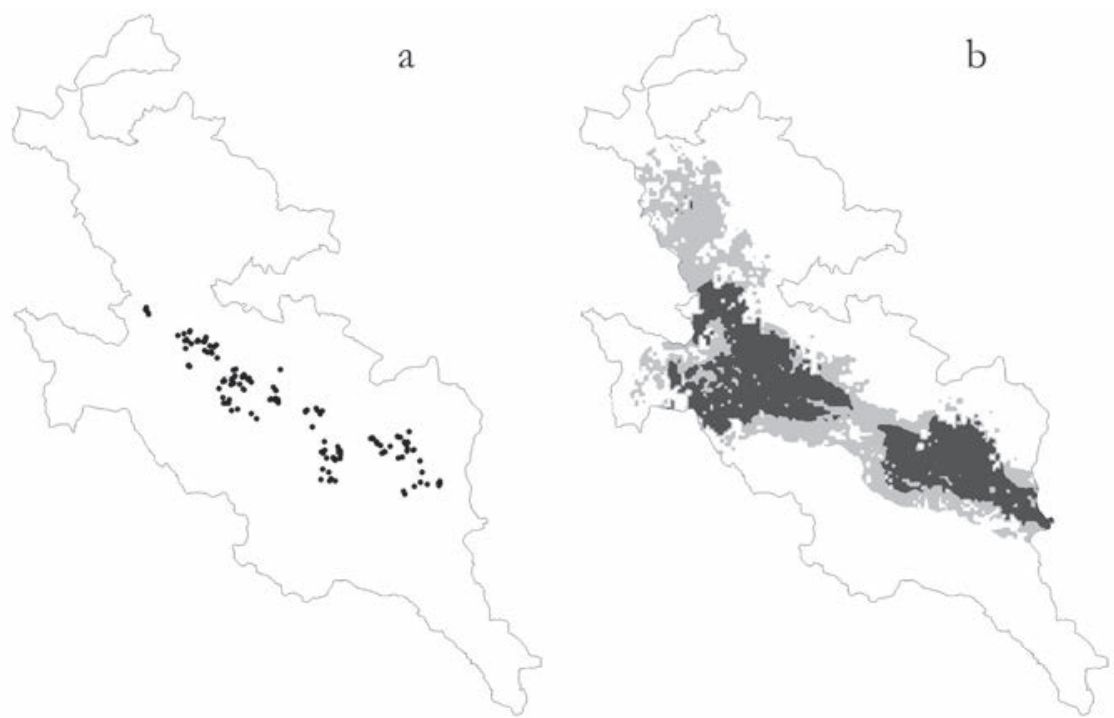

Fig. 4 - Carte dei segni di presenza (a) e dell'idoneità ambientale (b) per il Picchio nero nel Parco Nazionale delle Foreste Casentinesi, i cui valori si riferiscono al solo modello costruito con i soli segni di presenza; è riportato il confine dell'area protetta.

Fig. 4 - Maps of the indirect presence data (a) and of habitat suitability (b) for the Black Woodpecker in the Foreste Casentinesi National Park; values are relative only to the model built with indirect presence data. The National Park border is also reported.

L'esplicitazione dei modelli a livello peninsulare individua sostanzialmente tre aree ad elevata idoneità ambientale: l'Appennino settentrionale, praticamente per intero, parte dell'Appennino centrale, in corrispondenza dei maggiori rilievi dell'Abruzzo e del Lazio, e i rilievi della Sila (Figure 5 e 6).

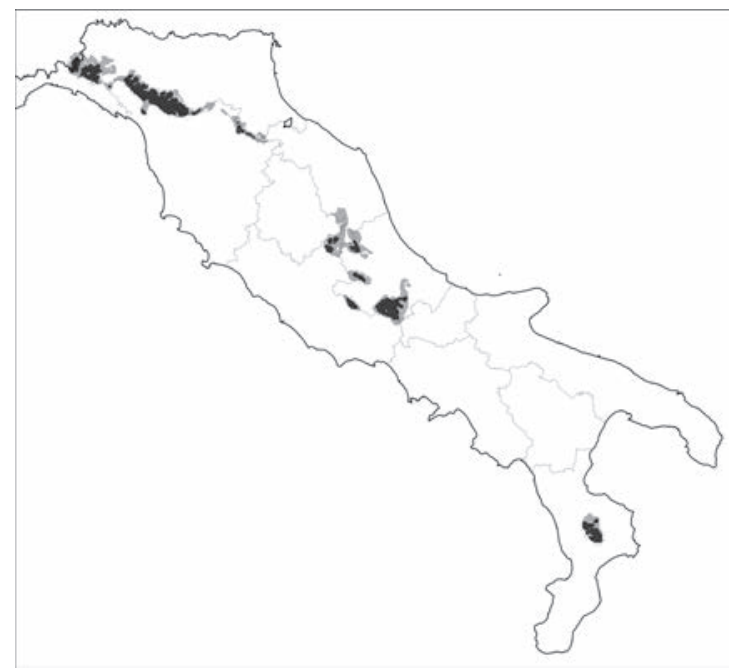

Fig. 5 - Esplicitazione a livello peninsulare del modello delle osservazioni. Le aree in nero sono quelle a più elevata idoneità, definite mediante la soglia MTP (Minimum Training Presence); quelle in grigio a minore idoneità, individuate con la soglia MTSS (Maximum Training Sensitivity plus Specificity).

Fig. 5 - Map showing the result of projecting the black woodpeckers model, only that one build with the observations, on the peninsular Italy. Black areas are the most suitable, identified by the MTP (Minimum Training Presence) threshold; the grey ones are less suitable and are identified using the MTSS (Maximum Training Sensitivity plus Specificity) threshold. 


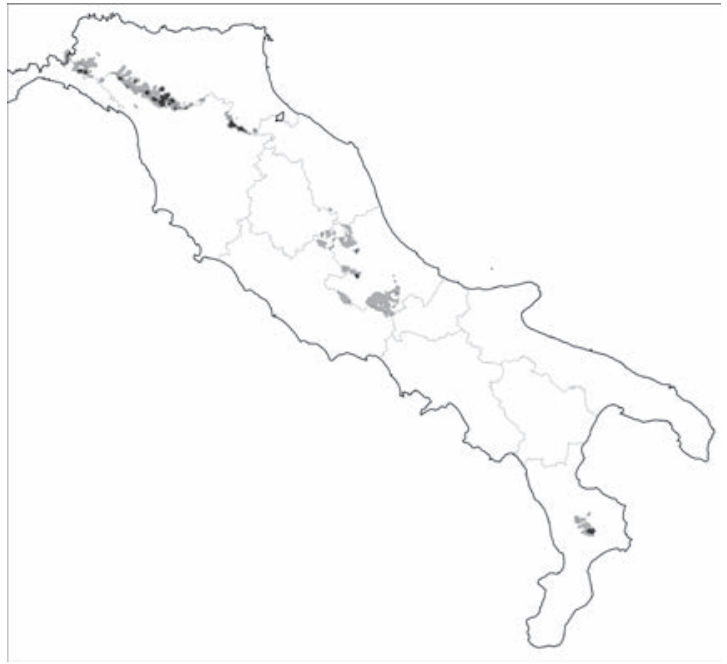

Fig. 6 - Esplicitazione a livello peninsulare del modello dei segni. Le aree in nero sono quelle a più elevata idoneità, definite mediante la soglia MTP (Minimum Training Presence); quelle in grigio a minore idoneità, individuate con la soglia MTSS (Maximum Training Sensitivity plus Specificity).

Fig. 6 - Map showing the result of projecting the black woodpeckers model, only that one build with the indirect presence data, on the peninsular Italy. Black areas are the most suitable, identified by the MTP (Minimum Training Presence) threshold; the grey ones are less suitable and are identified using the MTSS (Maximum Training Sensitivity plus Specificity) threshold.

\section{Discussione}

Analisi ecologiche

Gli elevati valori di AUC indicano come i modelli siano tutti molto efficienti e la coerenza ecologica dei fattori che maggiormente contribuiscono alla definizione delle aree idonee fanno ritenere i risultati affidabili. In sintesi, i modelli evidenziano un effetto consistente dei parametri climatici che concorrono entrambi ad individuare aree caratterizzate da condizioni decisamente mesofile, una relazione positiva con i boschi di abete bianco, che nel Parco è la conifera decisamente più diffusa, e una relazione positiva esiste anche con la faggeta, per la quale però un effetto soglia sembrerebbe indicare che la specie evita boschi puri di faggio.

I boschi di conifere montane e le faggete sono in effetti gli ambienti che la specie frequenta in maggior misura in Italia (BRICHETTI \& FraCASSO, 2007); i risultati ottenuti sono in linea anche con quanto recentemente messo in evidenza in ambiente alpino (BocCA et alii, 2007; ColPI et alii, 2009; PIROvANo, 2009) e anche in altre aree montane europee (TOBALSKE \& TOBALSKE, 1999). La preferenza per le conifere, che nei modelli hanno sempre un contributo maggiore rispetto alla faggeta per la quale è inoltre evidente, come già sottolineato, un effetto soglia, risulta anche sulle Alpi dove la faggeta è comunque utilizzata (ColPI et alii, 2009) e, anche se la faggeta non è selezionata positivamente, spesso i nidi sono scavati preferibilmente proprio nei faggi (BocCA et alii, 2007) come del resto accade anche nel Parco (CECCARELLI et alii, 2008). In altri paesi europei il picchio nero è capace di colonizzare differenti tipologie forestali (SPITZNAGEL, 1990), come ad esempio ambienti forestali ripariali a dominanza di Popolus sp. o anche pioppeti d'impianto (Gorman, 2004) e la decisa preferenza, nel territorio italiano, per i boschi 
di conifere montane e le faggete è probabilmente legata, almeno in parte, a fattori di tipo climatico (cui la specie sembra del resto rispondere anche nei nostri modelli) nel senso che altre tipologie forestali mancano da gran parte dei territori, come la pianura Padana, che sarebbero caratterizzati dalle condizioni climatiche più idonee alla specie. A conferma di questa ipotesi nell'Italia settentrionale la specie è stata recentemente osservata in periodo riproduttivo in tipologie forestali differenti da faggete o boschi di conifere montane, in alcune aree collinari e di pianura (MARTIGNAGO et alii, 2001; Utmar \& Padovan, 2005; Aimassi \& Reteuna, 2007; Gagliardi et alii, 2007).

Per quanto riguarda l'idoneità per condizioni decisamente mesofile che emerge dai modelli, questa deriva dal fatto che la specie è diffusa attualmente soprattutto nel versante romagnolo del Parco (CECCARELLI et alii, 2008) dove le condizioni climatiche sono generalmente più rigide rispetto al versante toscano. L'effetto del termotipo in particolare è marcato soprattutto nel modello per i segni, concentrati tutti nel versante romagnolo del Parco (CECCARELli et alii, 2008). Anche in questo caso però le variabili considerate potrebbero mascherare, in parte, fattori di altro tipo che condizionano l'attuale distribuzione della specie. La preponderante presenza della specie nel versante romagnolo potrebbe essere infatti dovuta al fatto che qui è iniziata la recente colonizzazione del Parco (CECCARELLi et alii, 2003) proseguita poi, nelle prime fasi, soprattutto nelle aree contermini (CECCARELLI et alii, 2008). Inoltre nel versante romagnolo le foreste in generale sono state nei decenni passati "meno gestite" (PADUlA, 1983) e sono più diffusi boschi maturi e stratificati (come la riserva di Sasso Fratino; BотTACCI, 2009), condizioni in generale più idonee per il Picchio nero. A questo proposito la mancanza di dati relativi alla struttura del bosco non permette considerazioni in merito a preferenze ecologiche a scala fine, anche se è noto che la specie, pur privilegiando boschi ben strutturati, con alberi di grosse dimensioni e ricchi di alberi morti o senescenti, mostra comunque una elevata plasticità ecologica (Mikusinski et alii, 2001) che sarebbe alla base della sua notevole capacità dispersiva e di colonizzazione (Mikusinski, 1995).

Le cartine di idoneità ambientale elaborate per l'area del Parco mostrano comunque la possibilità di una ulteriore espansione della specie, soprattutto nel versante toscano dell'area protetta. La maggiore attività selvicolturale, che qui si esercita rispetto alla porzione romagnola, peraltro ormai in larghissima parte condotta con criteri attenti alle esigenze della conservazione, non dovrebbe costituire un ostacolo alla diffusione della specie che, in altri contesti europei, frequenta e si riproduce abitualmente anche in foreste sottoposte a taglio, purché venga garantita la presenza di alberi, anche in numero limitato, abbastanza grossi da poter ospitare il nido (RoLSTAD et alii, 2000). Del resto, proprio nel versante toscano, i trend di crescita che hanno interessato, in tempi recenti, praticamente tutte le specie di uccelli legate al bosco, comprese quelle più esigenti, testimonia 
dell'evoluzione delle foreste, anche in quest'area, verso formazioni più mature e diversificate (TELlini Florenzano, 2004), dunque potenzialmente più idonee anche per il picchio nero.

L'esplicitazione a livello peninsulare dei modelli offre elementi interessanti per l'interpretazione della possibile origine della popolazione delle Foreste Casentinesi; in particolare le cartine sembrano avvalorare l'ipotesi, già avanzata da CECCARELLI et alii (2008), e recentemente confermata da analisi genetiche condotte da AlBERTI (2009), dell'origine alpina dei picchi delle Foreste Casentinesi. Gran parte dell'Appennino settentrionale infatti è caratterizzato da valori di idoneità alti, costituendo di fatto un ponte verso la Liguria e le Alpi marittime. Ad ulteriore conferma di questa ipotesi e anche della capacità predittiva del modello, in quest'area ad elevata idoneità, ad occidente dell'areale noto per la specie, si collocano alcune recentissime segnalazioni nel Parco Naturale Regionale dell'Aveto, nella porzione orientale dell'Appennino ligure (BAGHINO, 2009) e nell'Appennino bolognese (M. Zenatello, com. pers.) oltre ad una osservazione risalente al 1984 per il modenese (BERTARELLI, riportata in CECCARELLI et alii, 2008). È quindi probabile, o comunque plausibile, che individui in dispersione muovendosi verso oriente lungo l'Appennino abbiano trovato nelle Foreste Casentinesi un habitat particolarmente adatto, piuttosto isolato da altre aree idonee, e qui si siano stabiliti.

La netta preferenza per l'abete evidenziata dal modello, è inoltre un fattore in comune con le popolazioni alpine, anch'esse legate prevalentemente alle conifere (Bocca et alii, 2007) mentre altrove la specie frequenta anche differenti tipologie forestali e, ad esempio nell'Appennino meridionale, si trova spesso anche in faggete pure (BRICHETTI \& FRACASSO, 2007).

Riguardo le popolazioni di picchio nero dell'Appennino meridionale, è interessante notare come l'areale individuato a livello peninsulare dai modelli si sovrapponga solo parzialmente a quello conosciuto (BRICHETTI \& FrACASSO, 2007). I risultati dei modelli sono sostanzialmente condizionati dall'effetto delle variabili climatiche, ed in particolare dell'ombrotipo (cioè dall'umidità), che, in queste aree, raggiunge valori paragonabili a quelli dell'Appennino settentrionale solo nei rilievi della Sila. La presenza in aree anche differenti da quelle individuate dai modelli sembrerebbe indicare, e in un certo senso confermare (cfr. ALBERTI, 2009) un certo grado di specializzazione ecologica della popolazione dell'Appennino meridionale in cui potrebbe anche essere inclusa la preferenza per le faggete. A questo proposito si può aggiungere che, oltre alla scarsa diffusione delle conifere, con l'eccezione della Sila e dell'Aspromonte (Del Favero, 2008), nell'Appennino meridionale i boschi di latifoglie ed in particolare le faggete, per motivi che dipendono da complesse vicende anche economiche e sociali, hanno conservato nei decenni passati, almeno considerando il fenomeno a scala vasta, caratteristiche di maggiore idoneità (in sostanza più alberi di grosse dimensioni) rispetto a quelle dell'Appennino setten- 
trionale (HofFmanN, 1991) che possono aver favorito questi adattamenti.

I modelli sono in grado di mettere in luce in maniera piuttosto evidente le principali caratteristiche ecologiche della specie, coerenti con le conoscenze note per l'Italia (BRICHETTI \& FraCASSO, 2007) e, partendo da dati comunque molto localizzati forniscono utili elementi anche nell'interpretazione di fenomeni a scala più vasta. MaxEnt costruisce modelli molto efficienti anche con variabili piuttosto grossolane come quelle derivanti dalla CORINE o da banche dati climatiche a scala vasta come quelle utilizzate ma è anche in grado di apprezzare, qualora disponibili, elementi di maggiore dettaglio, come quelli derivanti dalla carta della vegetazione nel territorio del Parco, incrementando ulteriormente il grado di accuratezza delle previsioni (Tabella II). La mancanza di informazioni più dettagliate, ad esempio sulla struttura del bosco, non ha permesso di affinare ulteriormente lo studio né di estendere a scala di penisola i modelli più efficienti ma la capacità di funzionare praticamente con tutti i dati di presenza della specie, indipendentemente da metodi usati per raccoglierli, dalla loro distribuzione, dall'intensità di campionamento, si conferma una caratteristica molto utile del programma che trova il limite principale nella disponibilità di banche dati che forniscano, per quanto riguarda le variabili ambientali, informazioni omogenee per tutto il territorio che interessa indagare.

\section{Note all'utilizzo del metodo}

Le possibilità che il programma offre di impiegare diversi tipi di relazione tra la variabile dipendente e i parametri ambientali di cui si vuole testare l'effetto rende necessario, per un suo proficuo impiego, una valutazione critica delle stesse. A titolo di esempio riportiamo il caso della relazione esistente tra la variabile Fgt2000 e i segni di presenza in tre modelli costruiti variando le tipologie di features e il valore del coefficiente di regolazione; la descrizione dei tre modelli è riportata in Tabella III.

Tab. III - Confronto delle diverse combinazioni di coefficienti di regolazione e features utilizzati per la costruzione del modello dei segni di presenza. Viene indicato il valore del Coefficiente di Regolazione (C. Reg.), i diversi tipi di features utilizzati (lineare, quadratica, prodotto, soglia e lineare costante; vedi testo per eventuali spiegazioni) e il valore AUC.

La colonna Figura rimanda all'immagine della curva che esprime la relazione tra i segni di presenza e la variabile Fgt2000, scelta come esempio esplicativo.

Tab. III - Comparison among models build using different regularisation coefficient and features.

For each model the regularisation (C. Reg.) coefficient, the type of features (linear, quadratic, product, threshold, hinge) used and the AUC resulted are reported. The column "Figura" indicate the relative figure ( $n .7,8$ and 9) that shows the relationship between the presence of black woodpecker, here calculated only with indirect presence data, and the variable Fgt2000, chosen as an example.

\begin{tabular}{lcccccccc}
\hline modello & C. Reg. & Linear & Quadratic & Product & Threshold & Hinge & AUC & Figura \\
\hline Segni 1 & 1 & $\mathrm{x}$ & $\mathrm{x}$ & $\mathrm{x}$ & $\mathrm{x}$ & $\mathrm{x}$ & 0,946 \\
Segni 2 & 3 & $\mathrm{x}$ & $\mathrm{x}$ & $\mathrm{x}$ & $\mathrm{x}$ & $\mathrm{x}$ & 0,930 \\
Segni 3 & 3 & $\mathrm{x}$ & $\mathrm{x}$ & $\mathrm{x}$ & & & 0,904 \\
\hline
\end{tabular}


A ciascuno dei tre modelli è associata la curva che esprime la relazione esistente tra la variabile Fgt2000, scelta in questo caso come esempio, e la probabilità di trovare i segni di presenza (Figure 7, 8 e 9).
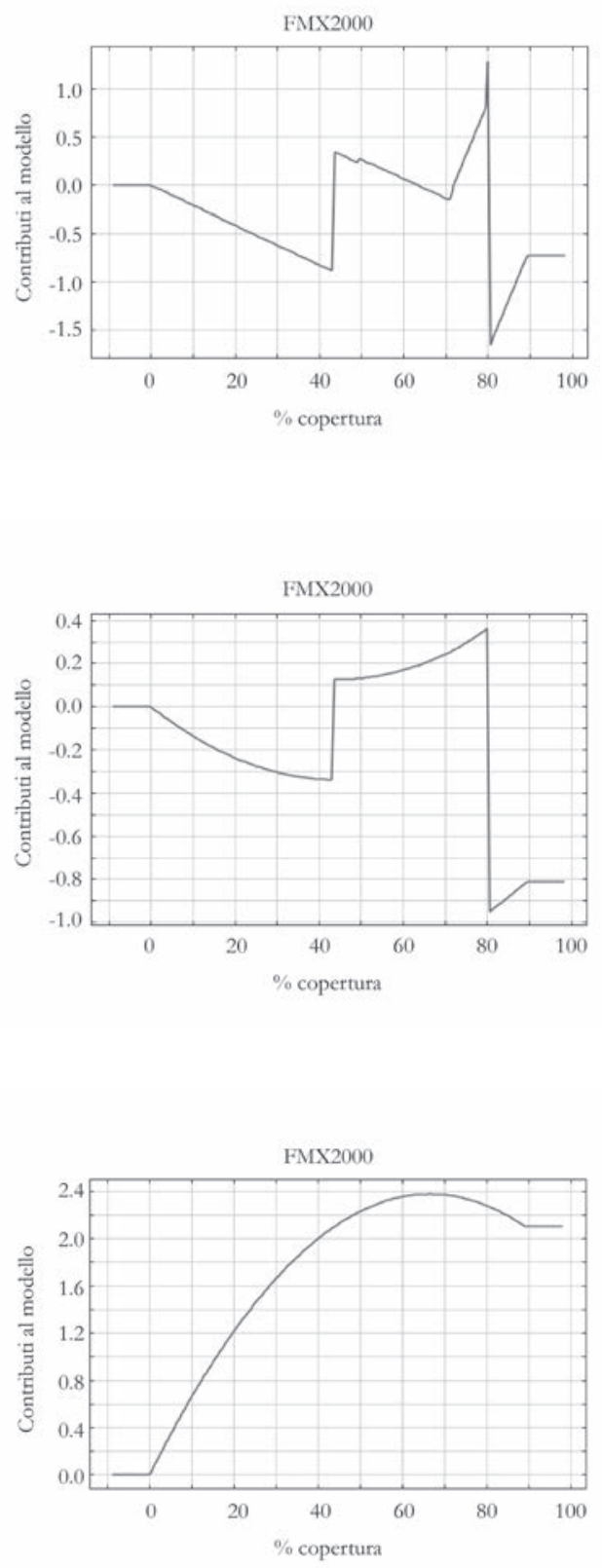

Fig. 7 - Andamento della relazione tra probabilità di presenza del Picchio nero, calcolata utilizzando solo i segni di presenza, e la copertura percentuale di faggeta col modello Segni 1 (C. Reg. = 1, tutte le features attive, cfr. Tabella III).

Fig. 7 - Relationship between the probability of Black Woodpecker presence, calculated only with the signs of presence, and the amount of beech forest in the model "Sign 1" (C. Reg. = 1, all the features activated, cfr. Table III).

Fig. 8 - Andamento della relazione tra probabilità di presenza del Picchio nero, calcolata utilizzando solo i segni di presenza, e la copertura percentuale di faggeta col modello Segni 2 (C. Reg. = 3, tutte le features attive, cfr. Tabella III).

Fig. 8 - Relationship between the probability of Black Woodpecker presence, calculated only with the signs of presence, and the amount of beech forest in the model "Sign 2" (C. Reg. = 3, all the features activated, cfr. Table III).

Fig. 9 - Andamento della relazione tra probabilità di presenza del Picchio nero, calcolata utilizzando solo i segni di presenza, e la copertura percentuale di faggeta col modello Segni 3 (C. Reg. = 3, escluse le features Threshold e Hinge, cfr. Tabella III).

Fig. 9 - Relationship between the probability of Black Woodpecker presence, calculated only with the signs of presence, and the amount of beech forest in the model "Sign 3" (C. Reg. = 3, all the features activated excluded the Threshold and Hinge ones, cfr. Table III). 
Analizzando l'andamento delle curve appare subito evidente come nei primi due casi il modello identifichi delle soglie difficilmente spiegabili, non coerenti da un punto di vista ecologico. Modificando le impostazioni del programma, scegliendo di utilizzare solo relazioni di tipo lineare, quadratico e prodotto (per una descrizione delle caratteristiche di tutte le relazioni vedi PHILLIPS \& Dudík, 2008), si ottiene invece una relazione curvilinea che identifica un massimo oltre il quale la presenza della faggeta sembra assumere un effetto negativo. Un risultato di questo tipo appare indubbiamente meglio spiegabile e soprattutto più coerente da un punto di vista ecologico, in linea, tra l'altro, con quanto emerso in altri studi (BoccA et alii, 2007). In questo caso, volutamente scelto come esempio esplicativo, appare subito evidente come relazioni come quelle ottenute nei primi due modelli siano facilmente ascrivibili ad artifici generati dal programma; tuttavia non è sempre così evidente, e in alcuni casi la presenza di un andamento più regolare, sempre ad esempio con delle soglie, può apparire effettivamente coerente.

\section{BIBLIOGRAFIA}

Agnelli P., Campedelli T., Cutini S., Farina F., Londi G. \& Tellini Florenzano G., 2009 - Algoritmi di massima entropia per la determinazione dell'idoneità ambientale a scala regionale di alcune specie di chirotteri. In: Dondini G., Fusco G., Martinoli A., Mucedda M., Russo D., Scotti M. \& Vergari S. (editors). Chirotteri italiani: stato delle conoscenze e problemi di conservazione. Atti del Secondo Convegno Italiano sui Chirotteri. Serra San Quirico (Ancona), 21-23 novembre 2008 - Parco Regionale della Gola della Rossa e di Frasassi, pp. 107-110.

Alberti D., 2009 - Caratterizzazione genetica delle popolazioni italiane di picchio nero - Tesi di Laurea. Corso di Laurea Specialistica in Conservazione e Gestione del Patrimonio Naturale. Facoltà di Scienze Matematiche, Fisiche e Naturali. Università di Bologna.

Aimassi G. \& Reteuna D. (editors), 2007 - Uccelli nidificanti in Piemonte e Valle d'Aosta. Aggiornamento sulla distribuzione di 120 specie. Memorie dell'Associazione Naturalistica Piemontese, Vol. VII - Associazione Naturalistica Piemontese, Bra (CN).

Baghino L., 2009 - Primi dati della comunità ornitica nidificante in tre foreste regionali del Parco Naturale dell'Aveto (GE) - Avocetta, 33 (1): 114-116.

Blasi C., Ciancio O., Iovino F., Marchetti M., Michetti L., Di Marzio P., Ercole S. \& Anzelotti I., 2004 - Il contributo delle conoscenze fitoclimatiche e vegetazionali nella definizione della rete ecologica d'Italia. In: Blasi C., D’Antoni S., Dupré E. \& La Posta A. (editors). Atti del Convegno "La conoscenza botanica e zoologica in Italia: dagli inventari al monitoraggio" - Quad. Cons. Natura, 18: 161-180.

Bocca M., CARisio L. \& Rolando A., 2007 - Habitat use, home ranges and census techniques in the Black Woodpecker Dryocopus martius in the Alps - Ardea, 95 (1): 17-29.

Bombi P., Luiselli L., Capula M. \& Salvi D., 2009 - Predicting elusiveness: potential distribution model of the Southern smooth snake, Coronella girondica, in Italy - Acta Herpetologica, 4 (1): 7-13.

Bordignon L., 2004 - Gli Uccelli della Provincia di Novara. Provincia di Novara - Tipolitografia di Borgosesia, Borgosesia (VC).

Bossard M., Feranec J. \& Otahel J., 2000 - Technical report no. 40. CORINE land cover technical guide. Addendum 2000 - European Environment Agency, Bruxelles.

Воттассі A. (editor), 2009 - La Riserva Naturale Integrale di Sasso Fratino: 1959-2009. 50 anni di conservazione della biodiversità - CFS/UTB, Pratovecchio.

Brichetti P. \& Fracasso G., 2007 - Ornitologia Italiana. Apodidae - Prunellidae - Alberto Per- 


\section{L'ECOLOGIA DEL PICCHIO NERO, DRYOCOPUS MARTIUS, NELLE FORESTE CASENTINESI}

disa Editore, Bologna.

Brotons L., Thuiller W., Araúso M.B. \& Hirzel A.H., 2004 - Presence-absence versus presence-only modelling methods for predicting bird habitat suitability - Ecography, 27: 437-448.

Burfield I. \& Van Bommel F. (editors), 2004 - Birds in Europe. Population estimates, trends and conservation status. BirdLife Conservation Series, no. 12 - BirdLife International, Cambridge.

Burgman M., Lindenmayer D.B. \& Elith J., 2005 - Managing landscapes for conservation under uncertainty - Ecology, 86: 2007-2017.

Campedelli T., Tellini Florenzano G., De Carli E., Buvoli L. \& Londi G., 2009 - Relazioni tra agricoltura e passeri nel paesaggio italiano, desunte dal progetto MITO 2000 - Ecologia Urbana, 21 (1): 21-25.

Ceccarelli P.P., 2011 - Picchio nero Dryocopus martius. In: Ceccarelli P.P. \& Gellini S. (editors). Atlante degli Uccelli Nidificanti nelle Provincie di Forli-Cesena e Ravenna (20042007) - S.T.E.R.N.A., Forli, pp. 190-191.

Ceccarelli P.P., Agostini N. \& Milandri M., 2003 - Osservazioni di Picchio nero, Dryocopus martius, nelle Foreste Casentinesi - Riv. ital. Orn., 73: 81-82.

Ceccarelli P.P., Agostini N., Milandri M. \& Bonora M., 2008 - Il picchio nero Dryocopus martius (Linnaeus, 1758) nel Parco Nazionale delle Foreste Casentinesi - Quad. Studi Nat. Romagna, 27: 143-154.

Colpi C., Varaschin M., Zenatello M. \& Luise R., 2009 - Selvicoltura ed avifauna sensibile. Il caso del picchio nero (Dryocopus martius) nel Parco Nazionale delle Dolomiti Bellunesi. In: Ciancio O. (a cura di). Atti del terzo Congresso Nazionale di Selvicoltura per il miglioramento e la conservazione dei boschi italiani - Taormina (MS), 16-19 ottobre 2008.

Cutini S., Bagni L., Campedelli T., Londi G. \& Tellini Florenzano G., 2009 - Ecologia e possibili linee di espansione della cincia dal ciuffo Lophophanes cristatus nell'Appennino - Alula, 16 (1-2): 329-334.

Del Favero R., 2008 - I Boschi delle regioni meridionali e insulari d'Italia. Tipologia, funzionamento, selvicoltura - CLEUP, Padova.

Elith J., Graham C.H., Anderson R.P., Dudík M., Ferrier S., Guisan A., Hijmans R.J., Huettmann F., Leathwick J.R., Lehmann A., Li J., Lohmann L.G., Loiselle B.A., Manion G., Moritz C., Nakamura M., Nakazawa Y., Overton J.M., Peterson A.T., Phillips S.J., Richardson K., Scachetti-Pereira R., Schapire R.E., Soberón J., Williams S., Wisz M.S. \& Zimmermann N.E., 2006 - Novel methods improve prediction of species' distributions from occurrence data - Ecography, 29: 129-151.

Favilli L., Piazzini A., Tellini Florenzano G., Londi G. \& Campedelli T., 2008 - Fattori ecologici a vasta scala che determinano la presenza di specie di ropaloceri di rilevante interesse in Toscana. In: Mairota P., Mininni M., Lafortezza R. \& Padoa Schioppa E. (editors), Ecologia e Governace del Paesaggio. Esperienze e prospettive-Atti del X Congresso Nazionale della Società Italiana di Ecologia del Paesaggio. Bari, 22-23 maggio 2008 - Università degli Studi di Bari, Politecnico di Bari, SIEP-IALE, pp. 257-264.

Ficetola G.F., Thuiller W. \& Padoa-Schioppa E., 2008 - From introduction to establishment of alien species: a preliminary analysis of bioclimatic differences between presence and reproduction localities in Trachemys scripta. In: CoRTI C. (editor). Herpetologia Sardiniae. $7^{\circ}$ Congresso Nazionale Societas Herpetologica Italica (Oristano, 1-5 ottobre 2008). Societas Herpetologica Italica - Edizioni Belvedere, Latina, pp. 266-269.

Ficetola G.F., Thuiller W. \& Miaud C., 2007 - Prediction and validation of the potential global distribution of a problematic alien invasive species - the American bullfrog - Diversity and Distributions, 13: 476-485.

Fielding A.H. \& Bell J.F., 1997 - A review of methods for the assessment of prediction errors in conservation presence/absence models - Environ. Cons., 24: 38-49.

Gagliardi A., Guenzani W., Preatoni D.G., Saporetti F. \& Tosi G., 2007 - Atlante Ornitologico Georeferenziato della provincia di Varese. Uccelli nidificanti 2003-2005 - Provincia di Varese; Civico Museo Insubrico di Storia Naturale di Induno Olona; Università degli Studi dell'Insubria, Varese.

Gorman G., 2004 - Woodpeckers of Europe. A study of the european Picidae - D \& N Publishing, Hungerford, Berkshire (UK). 
Gu W. \& Swihart R.K., 2004 - Absent or undetected? Effect of non-detection of species occurrence on wildlife-habitat models - Biol. Conserv., 116: 195-203.

Guisan A. \& Zimmerman N.E., 2000 - Predictive habitat distribution models in ecology - Ecol. Modell., 135: 147-186.

Guisan A., Graham C.H., Elith J. \& Huettmann F., 2007 - Sensitivity of predictive species distribution models to change in grain size - Diversity and Distributions, 13: 332-340.

Hernandez P.A., Graham C.H., Master M.M. \& Albert D., 2006 - The effect of sample size and species characteristics on performance of different species distribution modelling methods - Ecography, 29: 773-785.

Hofmann A., 1991 - Il faggio e le faggete in Italia. Collana Verde 81 - Corpo Forestale dello Stato, Roma.

Hosmer D.W. \& Lemenshow S., 2000 - Applied logistic regression. Second edition - J. Wiley \& Sons, New York.

Kerr J.T., Sugar A. \& Packer L., 2000 - Indicator taxa, rapid biodiversity assessment and nestedness in an endangered ecosystem - Conserv. Biol., 14: 1726-1734.

LARDELli R., 2009 - Ornitho.it. Una piattaforma comune per la raccolta e la condivisione di osservazioni e dati ornitologici in Italia - Avocetta, 33 (1): 142-146.

Liu C., Berry P.M., Dawson T.P. \& Pearson R.G., 2005 - Selecting thresholds of occurrence in the prediction of species distributions - Ecography, 28: 385-393.

Martignano G., Lombardo S. \& Mezzavilla F., 1992 - Considerazioni circa la diffusione e l'abbassamento altitudinale riscontrato nel Picchio nero (Dryocopus martius) - Boll. Cen. Orn. Veneto Or., 3: 23.

Martignano G., Silveri G. \& Mezzavilla F., 2001 - Diffusione e abbassamento altitudinale del Picchio nero Dryocopus martius in provincia di Treviso (Colli Asolani). In: Tellins Florenzano G., Barbagli F. \& Baccetti N. (a cura di). Atti del XI Convegno Italiano di Ornitologia, Castiglioncello (Livorno), 26-30 settembre 2001 - Avocetta, 25: 59.

Mezzavilla F. \& Bettiol K., 2007 - Nuovo Atlante degli Uccelli Nidificanti in Provincia di Treviso (2003-2006) - Associazione Faunisti Veneti.

Mezzavilla F. \& Stival E., 1996 - Rapporto Ornitologico Veneto Orientale - Anno 1996 - Boll. Cen. Orn. Veneto Or., 7: 1-13.

Mezzavilla F., 1989 - Atlante degli Uccelli nidificanti nelle province di Treviso e Belluno (Veneto) 1983-1988 - Mus. Civ. St. Nat. Montebelluna. D4 Industrie Grafiche, Casier.

Mezzavilla F., Stival E., Nardo A. \& Roccaforte P., 1999 - Rapporto Ornitologico Veneto Orientale - Anni 1991-1998 - Centro Ornitologico Veneto Orientale. Montebelluna.

Mikusinski G., 1995 - Population trends in black woodpecker in relation to changes and characteristics of European forests - Ecography, 18: 363-369.

Minusinski G., Gromadzki M. \& Chylarecki P., 2001 - Woodpeckers as indicator of forest bird diversity - Conserv. Biol., 15: 208-217.

MolLAnen A., 2002 - Implications of empirical data quality to metapopulation model parameter estimation and application - Oikos, 96: 516-530.

Padula M., 1983 - Storia delle Foreste demaniali Casentinesi nell'Appennino Tosco-Romagnolo. Collana verde 63 - Ministero Agricoltura e Foreste, Roma.

PAPE M. \& GAUBert P., 2007 - Modelling ecological niches from low numbers of occurrences: assessment of the conservation status of poorly known viverrids (Mammalia, Carnivora) across two continents - Diversity and Distributions, 13: 890-902.

Parodi R., 1999 - Gli uccelli della provincia di Gorizia - Comune di Udine, Edizioni del Museo Friuliano di Storia Naturale.

Pearson R.G., Raxworthy C.J., Nakamura M. \& Peterson A.T., 2007 - Predicting species distribution from small numbers of occurrence records: a test case using cryptic geckos in Madagascar - J. Biogeogr., 34: 102-117.

Pedrini P., 2010 - Picchio nero Dryocopus martius. In: Fornasari L., Londi G., Buvoli G., Tellini Florenzano G., La Gioia G., Pedrini P., Brichetti P. \& De Carli E., (eds). Distribuzione geografica e ambientale degli uccelli comuni nidificanti in Italia, 2000-2004 (dati del progetto MITO2000) - Avocetta, 34 (2): 37-38.

Peterson A.T., Pape M. \& Eaton M., 2007 - Transferability and model evaluation in ecological niche modelling: a comparison of GARP and Maxent - Ecography, 30: 550-560.

Phillips S.J. \& Dudík M., 2008 - Modelling of species distributions with Maxent: new exten- 
sions and a comprehensive evaluation - Ecography, 31: 161-175.

Phillips S.J., Anderson R.P. \& SchapiRe R.E., 2006 - Maximum entropy modelling of species geographic distribution - Ecol. Modell., 190: 231-259.

Pirovano A., 2009 - Il Picchio nero, un falegname per la conservazione della biodiversità nelle foreste alpine - Parco Orobie Valtellinesi, serie scientifica 3.

RAes N. \& Ter Steege H., 2007 - A null-model for significance testing of presence-only species distribution models - Ecography, 30: 727-736.

Rolstad J., Rolstad E. \& SAeteren O., 2000 - Black Woodpecker nest sites: characteristics, selection and reproductive success - J. Wildl. Manage., 64(4): 1053-1066.

Rushton S.P., ORMERod S.J. \& KeRBy G., 2004 - New paradigms for modelling species distributions? - J. Appl. Ecol., 41: 193-200.

Semenzato M. \& Amato A., 1998 - Comunità di uccelli nidificanti e svernanti nei boschi planiziali del Veneto centro - orientale (Italia N-E) - Lav. Soc. Ven. Sc. Nat., 23: 105-106.

Spitznagel A., 1990 - The influence of forest management on woodpeckers density and habitat use in foodplain forest of Upper Rhine Valley. In: Carlson A. \& Aulen G. (editors). Conservation and Management of Woodpecker Population, Report 17 - Dept. of Wildlife Ecology, Swedish University of Agricultural Sciences, Uppsala.

Tellini Florenzano G., 2004 - Birds as indicators of recent environmental changes in the Apennines (Foreste Casentinesi National Park, central Italy) - Ital. J. Zool., 71: 317-324.

Tellini Florenzano G., Campedelli T., Londi G., Dessì Fulgheri F. \& Gusmeroli E., 2008 - Idoneità ambientale a scala vasta per specie di interesse per la conservazione, ottenuta a partire da dati di sola presenza con algoritmi di massima entropia (MaxEnt). In: MAIrota P., Mininni M., Lafortezza R. \& Padoa Schioppa E. (eds). Ecologia e Governance del Paesaggio. Esperienze e prospettive - Atti del X Congresso Nazionale della Società Italiana di Ecologia del Paesaggio. Bari, 22-23 maggio 2008 - Università degli Studi di Bari, Politecnico di Bari, SIEP-IALE, pp. 237-244.

Toвalske C. \& Tobalske B.W., 1999 - Using atlas data to model the distribution of woodpecker species in the Jura, France - Condor, 101: 472-483.

Tofful M. \& Sponza S., 2007 - I picidi lungo il corso del fiume Isonzo - Book of Abstract del XIV Convegno Italiano di Ornitologia, Trieste, 26-30 settembre 2007: 56.

Tofful M. \& Sponza S., 2010 - I picidi lungo il corso del fiume Isonzo: analisi quantitativa e scelta del sito di nidificazione - Avocetta, 34 (1): 35-43.

Tsoar A., Allouche O., Steinitz O., Rotem D. \& Kadmon R., 2007 - A comparative evaluation of presence-only methods for modelling species distribution - Diversity and Distributions, 13: 397-405.

Utmar P. \& Padovan P., 2005 - Il Picchio nero, Dryocopus martius, nidificante in pianura nel Friuli Venezia Giulia - Riv. ital. Orn., 75 (1): 62-64.

Utmar P., 2003 - Svernamento di Picchio nero Dryocopus martius in un'area golenale di pianura in provincia di Gorizia. In: Conti P., Rubolini D., Galeotti P., Milone M. \& De Filippo G. (editors). Atti del XII Convegno Italiano di Ornitologio, Ercolano (Napoli), 23-27 settembre 2003 - Avocetta, 27 (num. spec.): 53.

Viciani D. \& Agostini N., 2008 - La carta della vegetazione del Parco Nazionale delle Foreste Casentinesi, Monte Falterona e Campigna (Appennino Tosco-Romagnolo): note illustrative - Quad. Studi Nat. Romagna, 27: 97-134. 